\title{
GISLER, Monica, Göttliche Natur ? Formationen im Erdbebendiskurs der Schweiz des 18. Jahrhunderts
}

\author{
Marie Thébaud-Sorger
}

\section{OpenEdition}

Journals

Édition électronique

URL : http://journals.openedition.org/ifha/599

DOI : 10.4000/ifha.599

ISSN : 2198-8943

Éditeur

IFRA - Institut franco-allemand (sciences historiques et sociales)

Référence électronique

Marie Thébaud-Sorger, « GISLER, Monica, Göttliche Natur? Formationen im Erdbebendiskurs der Schweiz des 18. Jahrhunderts », Revue de l'IFHA [En ligne], Date de recension, mis en ligne le 01 janvier 2007, consulté le 22 septembre 2020. URL : http://journals.openedition.org/ifha/599 ; DOI : https://doi.org/ 10.4000/ifha.599

Ce document a été généré automatiquement le 22 septembre 2020.

(C)IFHA 


\title{
GISLER, Monica, Göttliche Natur? Formationen im Erdbebendiskurs der Schweiz des 18. Jahrhunderts
}

\author{
Marie Thébaud-Sorger
}

L'étude de Monica Gisler se déploie à l'articulation d'une question globale portant sur la sécularisation des sciences à la période moderne, et de l'échelle locale des débats des naturalistes suisses au XVIIIe s. autour des phénomènes sismiques. Le tremblement de terre est-il expression de la colère de Dieu ou manifestation de sa providence ? L'ouvrage s'inscrit dans le cadre de recherche très productif qui se déploie autour de l'histoire des tremblements de terres et de la question du risque naturel (Quenet, Favier), mais aussi plus largement dans le champ d'une histoire des sciences renouvelée ayant pointé l'émergence de nouveaux discours savants autour la physique expérimentale au tournant du XVIIe s. (Shapin et Shaffer). L'auteur, en analysant l'ensemble des discours faces à ces événements, en relation avec leurs contingences sociales, politiques et culturelles, présente cette philosophie naturelle non plus dans le cadre du laboratoire mais bien à l'épreuve d'un nouveau terrain d'expérience incarné par les montagnes.

L'étude, menée suivant une large chronologie entre 1700 et 1800, est découpée en sept chapitres construits autour de figures exemplaires, de Johann Jakob Scheuchzers à Horace Bénédict Saussure, mais aussi au travers de débats menés entre des naturalistes moins connus, notamment autour de la réception d'un événement majeur : le tremblement de terre de Lisbonne de 1755.

La Suisse est alors un ensemble politique, confessionnel et linguistique très hétérogène. Les villes marquées par le protestantisme luthérien constituent cependant des lieux moteurs de l'Aufklärung scientifique dont l'unité réside dans une pratique commune de l'observation des montagnes qui nourrit un réseau dense de correspondances et de périodiques. Les amateurs abordent alors la géologie de manière latérale dans une démarche pragmatique fondée sur la collecte d'informations. Pour les naturalistes luthériens comme Scheuchzers, il s'agit par des méthodes expérimentales et 
instrumentales nouvelles de faire de la physique une expression de la providence divine. Ainsi sont réunies les conditions d'une " physico-théologie " très productive : l'histoire naturelle des montagnes devient le cadre d'une théologie appliquée à l'explication de phénomène naturel comme le tremblement de terre. Dans la deuxième moitié du XVIIIe s., on assiste à la progressive émergence d'un discours scientifique évacuant l'explication théologique. L'auteur scrute le rôle de la réception du séisme de Lisbonne et des théories de l'optimisme voltairien par les naturalistes suisses, notamment à la lumières de phénomènes sismiques très violents ayant lieu dans le Valais au même moment. Ces débats inaugurent les conditions d'un véritable tournant épistémologique. En effet, il n'existe pas de théorie consistante à proposer, et le cadre d'explication physico-théologique rencontre ici ses limites. Par sa recherche sur la formation des Alpes menée dès 1770, le travail de Saussure va permettre d'unifier la variété des observations par une explication du monde physique et une compréhension sécularisée des sciences. Il relie questionnement géologique et physique. Son hypothèse du rôle de l'électricité sur les phénomènes sismiques fondée sur l'étude de la composition de l'atmosphère (humidité, sécheresse) propose un tout autre cadre d'analyse, consolidant ainsi l'autonomie d'une nouvelle discipline.

Au-delà de la question critique de l'histoire des tremblements de terre pour lequel l'ouvrage présente une bibliographie complète et actualisée, ainsi que des annexes, cet ouvrage très solide jette un regard précieux sur la formation particulière de l'Aufklärung en Suisse et sur l'émergence de nouveaux savoirs et pratiques passant de l'histoire naturelle à la géologie.

Marie THEBAUD-SORGER (École des Hautes Études en Sciences Sociales, Paris) 